\title{
Impacto de la educación ambiental sobre docentes y alumnos. Doble intervención educativa
}

\author{
Antonio Pérez-Mora \\ Grupo de Investigación CIBERIMAGINARIO-UCLM. Toledo. España. \\ 1fantonioperez@gmail.com \\ ORCID: https://orcid.org/0000-0003-4563-2653 \\ Felipe Gértrudix-Barrio \\ Grupo de Investigación CIBERIMAGINARIO-UCLM. Toledo. España. \\ felipe.gertrudix@uclm.es \\ ORCID: https://orcid.org/0000-0001-8093-5698
}

[Recibido: 18 Junio 2020. Revisado: 19 Octubre 2020. Aceptado: 21 Noviembre 2020]

Resumen: Ante el conflicto que plantea el calentamiento global y la sobreexplotación de los recursos materiales, la necesidad de plantear nuevas alternativas en el modelo de producción de cara a un futuro más sostenible se hace cada día más imprescindible. Esto hace indispensable que existan estrategias de educación ambiental efectivas para desarrollar, tanto en las generaciones presentes como en las futuras, un sentido de cuidado y respeto para el entorno. El presente trabajo tiene como objetivo general medir el grado de concienciación y sensibilización de docentes y alumnos de Educación Infantil y Primaria en torno a la Economía Circular (EC), de manera transversal a través de diferentes herramientas. La metodología utilizada se encuentra bajo el paradigma mixto cuantitativocualitativo; cuantitativa mediante el uso del cuestionario y cualitativa a través de la observación participante y el registro de observación. De los resultados se infiere un interés mayoritario por los problemas medioambientales, si bien el conocimiento de ciertos términos, como alfabetización ecosocial, es escaso, entre los docentes. Además, se ha demostrado que la educación ambiental resulta motivadora para los alumnos, ya que se refuerza el sentido del aula como conjunto.

Palabras clave: Educación ambiental; Economía circular; Formación adicional; Concienciación, Sensibilización.

Impact of environmental education on teachers and students. Double educational intervention

\begin{abstract}
Faced with the conflict posed by global warming and the overexploitation of material resources, the need to propose new alternatives in the model of production for a more sustainable future is becoming more and more essential. This makes it essential that effective environmental education strategies exist to develop in both present and future generations a sense of care and respect for the environment. The present work seeks as a general objective to measure the degree of awareness and sensitization of teachers and students of kindergarten and primary education around the Circular Economy (CE), in a transversal way through different tools. The methodology that has been carried out is under the mixed quantitative-qualitative paradigm; quantitative through the use of the questionnaire and qualitative through participant observation and observation recording. From the results, a majority interest in environmental problems can be inferred, although knowledge of certain terms is low among teachers, such as eco-social literacy. Furthermore, it has been shown that environmental education is motivating for students, as it reinforces the sense of the classroom.
\end{abstract}

Keywords: Environmental education; Circular economy; Additional training; Raising awareness; Sensitivity.

Para citar este artículo: Pérez-Mora, A. y Gértrudix-Barrio, F. (2020). Impacto de la educación ambiental sobre docentes y alumnos. Doble intervención educativa. Revista de Educación Ambiental y Sostenibilidad 2(2), 2302. doi: 10.25267/Rev_educ_ambient_sostenibilidad.2020.v2.i2.2302 


\section{Introducción}

Ante el conflicto que plantea el calentamiento global y la sobreexplotación de los recursos materiales, la necesidad de plantear nuevas alternativas en el modelo de producción de cara a un futuro más sostenible se hace cada día más imprescindible. Es por ello, que la investigación acerca del medioambiente y de cómo trasladar esos valores a la sociedad por medio de una educación responsable adquiere sentido en un contexto donde la comunidad educativa de los países vecinos evoluciona hacia una modernidad que no puede concebirse sin las respuestas a las preguntas que plantea la problemática medioambiental.

Ante la génesis de la Economía Circular (EC) y el estudio de sus posibilidades, beneficios y límites, la educación ha conseguido ver en ella un aliado donde desarrollar un camino conjunto por el que procurar la consecución de un planeta más limpio. Además, debido a la novedad del término, los trabajos a este respecto son escasos, por lo que se necesitan aportaciones que ayuden al resto de la comunidad a ir afianzando cimientos concretos en función de una bibliografía consolidada.

El presente trabajo tiene como objeto de estudio la EC y su relación con el ámbito escolar. Y, para ello, nos planteamos ciertas incógnitas como: ¿la educación ambiental constituye una preocupación en la programación de los docentes?, o ¿influye positivamente el trabajo sobre economía circular en la percepción infantil del medioambiente?

De esta forma se deriva un objetivo general como es el de medir el grado de concienciación y sensibilización de docentes y alumnos de Educación Infantil y Primaria en torno a la EC, de manera transversal a través de diferentes herramientas.

\section{Marco teórico}

\section{Marco histórico}

Respecto a la economía circular, encontramos que la comunidad educativa y científica lleva manejando el concepto de desarrollo sostenible durante décadas. Principalmente, los efectos del desarrollo socio-económico comenzaron a hacerse notar en el medioambiente a finales del siglo XX, por lo que la preocupación por su cuidado surge durante sus últimas décadas.

Con la Primera Cumbre de la Tierra en Estocolmo (Naciones Unidas, 1973), se produjo el primer acercamiento real a una preocupación internacional por el medio ambiente vinculado a cuestiones técnicas, demográficas y culturales (Boudes, 2011). Este primer acercamiento dio lugar a una legislación «blanda» sobre el medio ambiente y el inicio hacia nuevos modelos de actuación más «verdes». Las diferentes recomendaciones que se formularon concluyeron que la acción humana era a la vez causa y efecto del ecosistema en el que se desarrolla, de tal forma que señalaron «los límites de la racionalidad económica y los desafíos que genera la degradación ambiental al proyecto civilizatorio de la modernidad» (Leff, s.f.).

Una década después se creó la Comisión de Desarrollo y Medio Ambiente, donde expertos de todo el mundo coincidieron en la idea de que los hábitos de consumo debían cambiar para evitar que la destrucción del ecosistema llegase a ser irreversible 
(Ramírez-Treviño, Sánchez-Núñez y García-Camacho, 2004), y ofrecieron el famoso informe Brundtland (Toro-Sánchez, 2007).

A partir de este momento se abrieron nuevas líneas de investigación que dieron lugar a nuevos términos como «desarrollo sostenible». Por su parte, la Comunidad Europea fue una de las precursoras a nivel mundial en adoptar medidas para conseguir mejorar ese aspecto: la primera Directriz sobre Protección del Medio Ambiente y los Consumidores, el primer Programa de Acción Ambiental y, como consecuencia, el Libro Verde como táctica para moderar los residuos provenientes de combustibles fósiles (Comisión Europea, 2013).

Todas estas ideas están implícitas en el concepto de EC, que ya fue defendido por Pearce y Turner (1990) quienes demostraron el impacto que la economía de la época tenía sobre el medioambiente, analizando el modelo de economía lineal y sus efectos; y, Stahel y Reday (1981) entendieron la economía como un sistema circular con unos materiales que fluyen constantemente y, tratando de prevenir al máximo el gasto, creando nuevos empleos, mejorando la eficiencia e innovando en nuevas tecnologías, y con ello crean «nuevos modelos de negocio como la ecología industrial, la simbiosis territorial, la economía de la funcionalidad, la economía colaborativa, etc.» (Fundación para la Economía Circular, 2017, p. 5 ).

Pero armonizar las acciones humanas y el crecimiento económico con la protección del medio ambiente no es un reto fácil, ya que se debe romper con los paradigmas dominantes que más perjudican. La racionalidad ambiental pasa irremediablemente por ser integrada con la naturaleza (Leff, 2004).

\section{Economía circular: la necesidad del cambio}

\section{Principios de la economía circular}

Para lograr implantar un nuevo modelo económico es necesario conocer cuáles son sus ventajas respecto al actual y establecer líneas de actuación para conseguir un cambio. Así, se enfatiza la necesidad de apostar por una postura regenerativa y una postura eco-céntrica, para lo que se necesita actuar sobre aspectos concretos que impiden ese cambio de mentalidad. En líneas generales, Stahel y Reday-Mulvey (1977) teorizan acerca de reducir la circulación de recursos como medida para hacer los recursos más valiosos y eficientes, así como para minimizar el gasto potencial. Todo esto pasa por mejorar la calidad de los productos y la eficiencia en el proceso de venta y reciclaje.

Por tanto, se hace necesario establecer unos principios básicos que rijan la puesta en práctica de este modelo económico. Estos principios, según Morató et al. (2017) de la Fundación Cotec , son los siguientes:

- Diseño de la prevención de residuos.

- Construcción de resiliencia a través de la diversidad.

- Uso de energías renovables.

- Los residuos son comida.

- Pensamiento en sistemas.

- Pensamiento en cascadas.

- Enfoque en el rendimiento. 


\section{Posibles beneficios multidisciplinares}

Tras una primera toma de contacto con la EC y sus bases principales se han evidenciado ciertas ideas que pueden elevar las esperanzas en torno a un futuro sostenible bajo la utilización de dicho paradigma de consumo, el cual puede tener unos efectos muy beneficiosos que además se evidencian ya en cómo el reciclaje, que aún no se da en su mejor versión posible, afecta en múltiples ambientes.

- Impacto medioambiental

El reciclaje y la reutilización de recursos reportan una cantidad enorme de beneficios al medioambiente, reduciendo la emisión de gases de efecto invernadero, la contaminación del suelo, así como la contaminación del agua y del aire, ayudando a conservar y preservar los recursos naturales y generando empleo.

Además, debido a que la contaminación se da en multitud de ámbitos, un reciclaje cíclico y efectivo aboga por tratar el problema desde la raíz, que es, en muchos casos, la extracción de recursos. Mientras se consigue una transición hacia fuentes de energía renovables, ha de aumentarse el reciclaje de calidad en estos procesos, evitando la contaminación de aguas y suelos (Upadhayay y Alqassimi, 2019; Ghisellini y Ulgiati, 2019).

- Impacto económico

Como incentivo al modelo de EC y al reciclaje como recurso, el análisis sobre el impacto económico aporta un valor añadido a este proceso, pues puede atraer a grandes empresas y corporaciones, cuya actividad es también indispensable en la lucha contra el cambio climático.

El reciclaje emplea a un amplio abanico de sectores, desde la recolección, el transporte, la personalización, la separación, el procesado, la creación del segundo producto, la manufacturación, el empaquetado y el proceso de venta. Así, existen ejemplos concretos que estiman que las industrias relacionadas con el reciclaje crean en Estados Unidos más de un millón de trabajos que reportan más de cien millones de dólares de beneficio.

- Impacto social

El reciclaje se ha convertido en un aspecto global que permite generar comunidades y acciones de interacción social donde se fragua la conciencia ecológica y que además mejora los ambientes de vida y de trabajo de multitud de sectores, incrementa las acciones de voluntariado y promueve investigaciones culturales y científicas en todo el mundo.

\section{- Impacto educativo}

El estudio del reciclaje en todos los niveles educativos ha resultado ser una experiencia enriquecedora. El aumento del interés por aprender y mejorar en la competencia natural se ha evidenciado en los últimos años, puesto que este tipo de educación genera multidisciplinariedad entre campos como la ciencia, la química, la mecánica, la eléctrica, etc. No obstante, el beneficio va más allá del meramente educacional, puesto que en el trabajo de la importancia del reciclaje y los efectos del cambio climático trasciende una necesidad de cambio de paradigma que puede permitir una didáctica renovada acerca de un modelo alternativo como es la EC. 
Esta concienciación debe empezar en los primeros niveles, y en especial en Educación Infantil por su carácter globalizador del aprendizaje. En este sentido, ya existen diversas investigaciones que apuntan claramente a cómo ayudar a trabajar el reciclaje en el aula, creando una conciencia inicial en los ciudadanos que, en su mayoría, no han tenido experiencias externas similares, y que deberán lidiar con los efectos en un futuro a largo plazo virando hacia un modelo de EC (Buil, Roger-Loppacher y Tintoré, 2019; Boyd, 2020; Schoden et al., 2020).

\section{Hacia la transición a través de laeducación.}

Ante este encorsetamiento en el que se halla la transición hacia la EC (Irving-Olsen, (2019), la escuela puede tener la llave hacia un futuro sostenible en el que el modelo de consumo sea más respetuoso con el medio ambiente y más comprometido con el planeta (Pearson, 2019). De esta forma, el trabajo de este tipo de realidades en la educación puede ir formando a ciudadanos críticos con una conciencia ecológica que permita avanzar en el futuro. Para ello, es necesario apuntar que ya existen trabajos que han comenzado a profundizar en este primer eslabón de la cadena del cambio de paradigma, que han podido observar que existen algunos factores que entorpecen el desarrollo de la competencia medioambiental como son los horarios del profesorado o la falta de conocimiento sobre el tema. (Lee, 2000).

En el sistema educativo español, el diseño de los horarios desde $1^{\circ}$ de Primaria hasta el final de la Educación Secundaria Obligatoria crea la necesidad de plantear un proyecto interdisciplinar, pues no existe la flexibilidad necesaria para trabajarlo de manera holística, como sí permite, por ejemplo, el sistema de la Educación Infantil, que puede ser objeto de estudio por su versatilidad y capacidad del alumnado de concienciación y sensibilización.

Toda esta génesis teórica debe materializarse en un proceso de sensibilización ciudadana a través de la apropiación del concepto. Esto hace indispensable que existan estrategias de educación ambiental efectivas para desarrollar tanto en las generaciones presentes como en las futuras un sentido de cuidado y respeto para el entorno (Torres, 1996).

\section{Método}

\section{Objetivos}

El nexo entre la teoría y la práctica pasa por una concreción en la acción establecida sobre las bases de unas nociones concisas que faciliten la adaptación al contexto educativo. En este trabajo, pueden identificarse principalmente tres:

- El modelo económico actual es insostenible a largo plazo, puesto que sus bases se encuentran cimentadas sobre el uso de combustibles fósiles, que suponen un expolio continuo que está abocado a terminar.

- Para propiciar un cambio se postula la EC como alternativa donde alterar la cadena de producción, centrando la atención en el diseño elaborado de los materiales.

- La base de esta revolución se encuentra en la educación, puesto que es necesario transmitir el sentido de responsabilidad y sensibilización a todos los estratos de la sociedad, por lo que la docencia permite acceder a individuos de 
todas las clases socioeconómicas y cuyo futuro se verá afectado por los efectos del cambio climático.

La falta de trabajos acerca de la relación existente entre la EC y la educación, junto con la acuciante necesidad de una sensibilización en torno a la problemática medioambiental, crean el contexto en el que se enmarca este estudio.

Por tanto, y teniendo en cuenta que el objeto de estudio es el impacto de la educación ambiental y la EC, en particular, sobre docentes y alumnos, y que se busca como objetivo general (OG) medir el grado de concienciación y sensibilización de docentes y alumnos de Educación Infantil y Primaria en torno a la EC, de manera transversal a través de diferentes herramientas, se han planteado los siguientes objetivos específicos:

- 01. Conocer si existen diferencias en conocimiento y sensibilidad en torno a la EC, según la edad de los participantes.

- 02. Implementar una intervención didáctica en un aula de Educación Infantil en torno al reciclaje y la EC.

- 03. Evaluar el grado de concienciación entre los niños y niñas de Educación Infantil en torno al cuidado del medio ambiente mediante acciones educativas de reciclaje y economía circular.

\section{Planteamiento metodológico. Definición de conceptos}

La metodología que se ha llevado a cabo se encuentra bajo el paradigma mixto cuantitativo-cualitativo. El planteamiento cualitativo en educación aporta un punto de vista holístico, profundidad en los datos e interpretación de lo que se va captando activamente (Hernández, Fernández y Baptista, 2006). En nuestro caso se ha utilizado la observación participante, ya que los datos se recogen de modo sistemático y no intrusivo (Taylor y Bogdan, 1984), y con ello se pretende contestar a los objetivos específicos 2 y 3 del trabajo.

El enfoque cuantitativo se ha utilizado con el fin de establecer ciertos patrones de comportamiento y probar teorías siguiendo los postulados de Latorre, del Rincón y Arnal, (2003) y Albert (2007). En nuestro caso, se ha utilizado el cuestionario como instrumento cuantitativo, respondiendo, con ello, al objetivo general y objetivo específico 1.

Este trabajo se ha desarrollado en un centro público de Educación Infantil y Primaria situado en una en una localidad de la provincia de Toledo.

\section{Procedimientos}

La intervención educativa sigue una planificación temporal espaciada: los docentes acudieron a una jornada de formación en la que se les introdujo en la terminología de la educación ambiental y la EC. Posteriormente, tras un debate abierto, todos los participantes contestaron un cuestionario de sensibilización sobre los elementos de la EC y su impacto en el aula.

El procedimiento para la aplicación con los niños y niñas del aula ha sido gradual, puesto que el término EC se introduce a través de asambleas y actividades. Además, se ha debatido la utilización de papel albal como material perjudicial e innecesario para el medioambiente con los alumnos, cuyas ideas son valoradas, hasta llegar a concluir que es necesaria nuestra acción. Se propuso una actividad llamada «La bola que no 
mola», que consistía en que, durante el tiempo dedicado a la merienda, utilizábamos el papel albal para crear una esfera de este material que iba creciendo a medida que los alumnos traían ese tipo de envoltorio. El objetivo común de la clase era el de conseguir que esa pelota no aumentase con el paso de los días, por lo que iría calando la idea de sustituirlo por envoltorios reutilizables.

\section{Técnicas e instrumentos de recogida de información}

A lo largo del proceso de este trabajo se han utilizado distintas técnicas para conseguir tener un registro sistemático de los datos obtenidos.

En concreto, se han utilizado distintas tipologías como:

- Cuestionario transversal, basado en el que desarrollaron Sánchez-Emeterio y Figueira (2019) y al que se añadieron términos relacionados con la EC, para establecer una serie de estándares sobre el nivel de concienciación medioambiental de la propia acción de los docentes.

- Observación directa, como herramienta fundamental en un aula, donde varios acontecimientos suceden de manera simultánea y son la intuición del docente y su capacidad de análisis las que determinan los aprendizajes sintetizados por parte de los infantes (Rekalde, Vizcarra, y Macazaga, 2014).

- Registro de observación diario, utilizado para conseguir dejar constancia diaria de un fenómeno que se considera interesante a la hora de perseguir un objetivo didáctico o científico.

\section{Variables de investigación}

De las dos vertientes que se desprenden en la investigación podemos extraer distintas variables. En cuanto a la intervención con los docentes, la variable dependiente es el grado de concienciación de los docentes, que recibieron formación específica, mientras que la independiente es la respuesta en el aula que los maestros han llevado a cabo.

Desde el punto de vista de la intervención con los alumnos, la variable dependiente a medir es la utilización de papel albal, puesto que se trata de un material popular, cuyo proceso de reciclaje es costoso y su uso puede ser sustituido por cualquier envoltorio reutilizable y lavable.

En la misma línea, el objetivo de medir el impacto de la noción EC entre el alumnado se torna más complejo debido a lo abstracto de la propuesta. La variable independiente se trata del concepto en sí mismo, que será medible a través de la observación directa de los alumnos en las distintas actividades que se realizan mediante la intervención educativa, y será también importante el análisis documental para poder adaptarlo al nivel pertinente (Campos y Lule, 2012).

Tabla1. Relación entre las variables y las técnicas de investigación

\begin{tabular}{|l|l|}
\hline Variables de la investigación & Técnicas de investigación \\
\hline 1. Grado de concienciación de los docentes & Cuestionario transversal \\
\hline 2. Concepto «economía circular» & Análisis documental y observación directa \\
\hline 3. Utilización de papel albal & Registro de observación diario \\
\hline
\end{tabular}




\section{Población y muestra. Participantes en el estudio}

La población puede incluir cualquier elemento que influya en los sujetos que intervienen en el desarrollo del trabajo (de Canales, de Alvarado y Pineda 1994). Así, y debido al carácter dual de la investigación, la población de la intervención con los docentes son todos los maestros y maestras del centro educativo (38), tanto de Educación Infantil como de Educación Primaria, con una muestra aleatoria final de $\mathrm{n}=27$ docentes $(71 \%$ del total de la población). El conjunto de los docentes está compuesto por 5 hombres y 22 mujeres, de los que 9 dan clase en Educación Infantil, 14 en Educación Primaria y 4 son especialistas en ambos niveles. Además, la muestra se ha estratificado en cuatro grupos de edades (ver figura 1), si bien se ha realizado una agrupación simplificada en dos target de edad (los comprendidos entre 25 y 44 -subgrupo1- y entre 45 y 65 -subgrupo 2-)

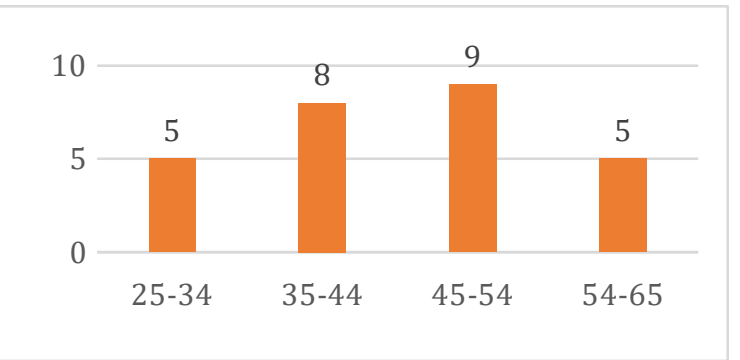

Figura 1. Muestra por edades de los participantes

En cuanto a la intervención con los alumnos, la población son todos los niños y niñas del segundo nivel del segundo ciclo de Educación Infantil, con una muestra de una sola aula, con un total de $n=21$ alumnos, de los que 11 son niñas y 10 niños, 3 de estos son inmigrantes.

\section{Cronograma de la intervención}

La propuesta didáctica se dividió en dos intervenciones: una a nivel de claustro y otra a nivel de aula.

Intervención a nivel de claustro:

- Descripción: Jornada de introducción a la terminología específica con un debate abierto final.

- Temporalización: el 14 de febrero de 2020.

- Objetivo: Recoger los datos que sirviesen de aproximación a los conocimientos y nivel de sensibilización de los docentes en torno a la economía circular.

- Sistema de evaluación: Cuestionario al finalizar la jornada.

Intervención a nivel de aula:

- Descripción: Se llevó a cabo una introducción previa de la terminología y la problemática medioambiental a través de asambleas y conversaciones con los alumnos. Tras esto, se llevó a cabo la actividad denominada «La bola que no mola», donde se registraba si los niños traían un envoltorio reutilizable o un envoltorio de papel albal.

- Temporalización: El trabajo de introducción terminológica comenzó el 4 de marzo y fue progresivo, mientras que el registro de observación comenzó el 17 
de febrero de 2020, extendiéndose en 16 jornadas escolares, hasta el 11 de marzo de 2020.

- Objetivo: comprobar si el trabajo previo de la educación ambiental podía tener un impacto real en la conducta de los alumnos, a través de la observación de un hábito muy común para ellos como es el de traer el aperitivo del recreo en un tipo de envoltorio que era predominantemente no reutilizable (papel albal).

- Sistema de evaluación: Registro de observación diario, disponible en el Anexo 2.

\section{Análisis de resultados}

\section{Análisis de resultados de intervención con docentes}

El cuestionario se elaboró en tres módulos distintos (Anexo 1). En el módulo A se recogieron datos relativos a la información del individuo acerca de sus estudios, su edad y su formación. Por otro lado, en el módulo B se analizan sus conocimientos acerca de distintos conceptos relacionados con la educación medioambiental, mientras que en el módulo $\mathrm{C}$ se evalúan los hábitos de reciclaje y de respeto hacia el medioambiente que los docentes llevan a cabo tanto en su vida personal como con sus alumnos en su vida académica.

De esta manera, en los dos últimos módulos del cuestionario pueden tomarse referencias acerca de distintos conceptos o costumbres. Esta información podrá ser de utilidad de manera autónoma, así como podrá dar lugar a cruces con el módulo A, donde cierta información personal puede arrojar luz acerca de características específicas en relación con los elementos baremados.

Los sujetos se muestran de manera anónima y se apilan en función de los grupos de respuestas dadas, ponderadas en una escala de Likert de 1 a 5 , que arrojaron los siguientes datos:

\section{Módulo B}

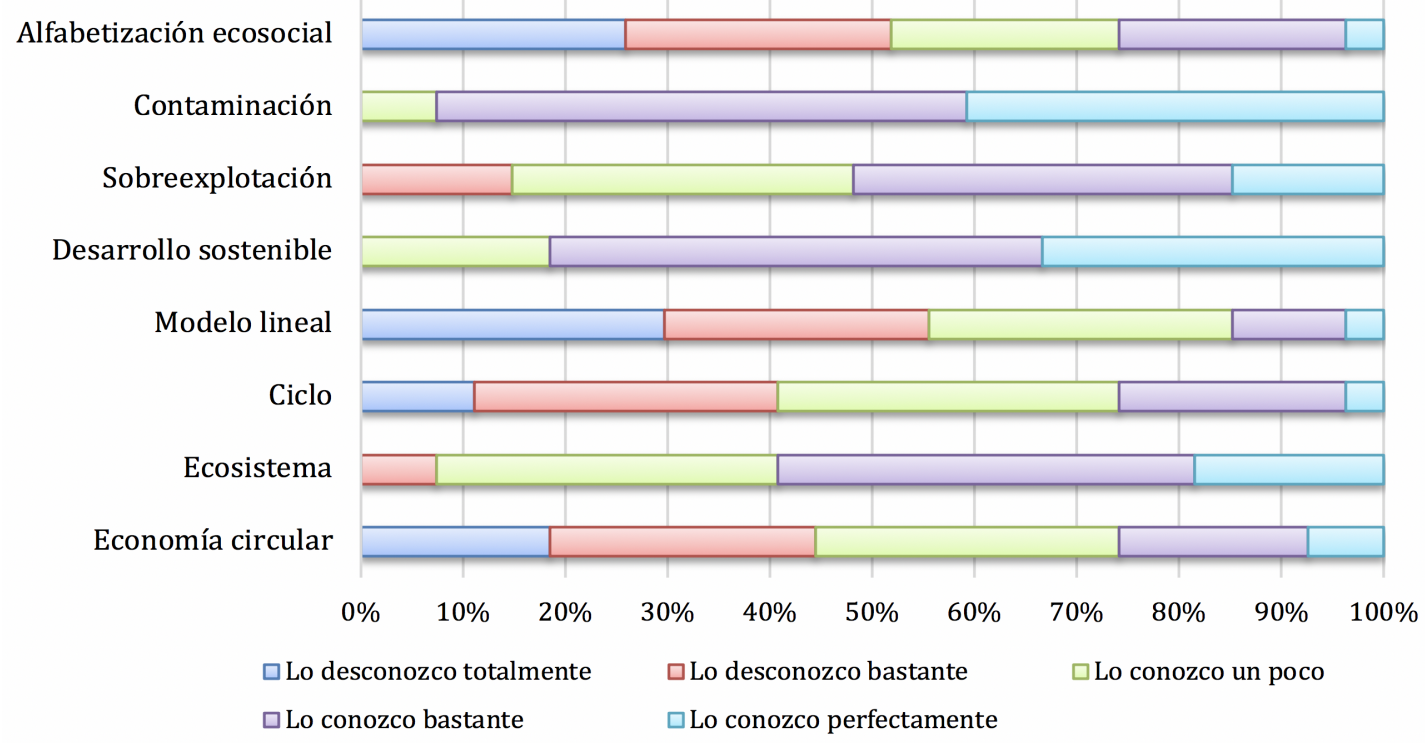

Figura 2. Evaluación de conceptos sobre educación medioambiental 
En lo referente a la Figura 2, el módulo B establece los conocimientos previos de los docentes en lo que a términos de educación ambiental respecta. Así, a simple vista puede observarse cómo los términos más generales son altamente populares a través de la anchura de las franjas de colores azul claro y morado. De esta forma, «contaminación» o «desarrollo sostenible» consiguen las tasas más altas, junto con «ecosistema». Este resultado es lógico y esperable, dado que se trata de generalizaciones muy comunes y utilizadas en medios de comunicación y otras fuentes de información.

Los tres términos obtienen puntuaciones muy similares, con una media de 3,6 puntos de diferencia, ya que «contaminación» tiene 55 puntos (subgrupo 1) frente a 61 (subgrupo 2), «desarrollo sostenible» 57 frente a 54 y «ecosistema» obtiene 49 frente a 51 puntos.

Sin embargo, existen otros términos más concretos cuya casuística es totalmente distinta. Estos son aquellos que son más específicos. Así, podemos apreciar que el término «alfabetización ecosocial» es uno de los términos menos conocidos, y, además, si dividimos a los participantes en dos grupos, observamos cómo el primer grupo, cuya edad es mayor, solo representa el 30\% del conocimiento de este término, mientras que es el grupo de los docentes más jóvenes el que acapara el 70\% restante.

En esta misma línea, el concepto «modelo lineal», que es el término más desconocido para el conjunto, presenta una división similar donde el primer subgrupo obtiene un porcentaje similar, del 35\%, ya que acapara 21 puntos sobre el total de sumas de las respuestas, que es 60 .

Por lo tanto, una vez que se han evidenciado los casos más particulares de esta diferencia, pueden analizarse todos los casos para tratar de confirmar o desmentir una tendencia a este respecto.

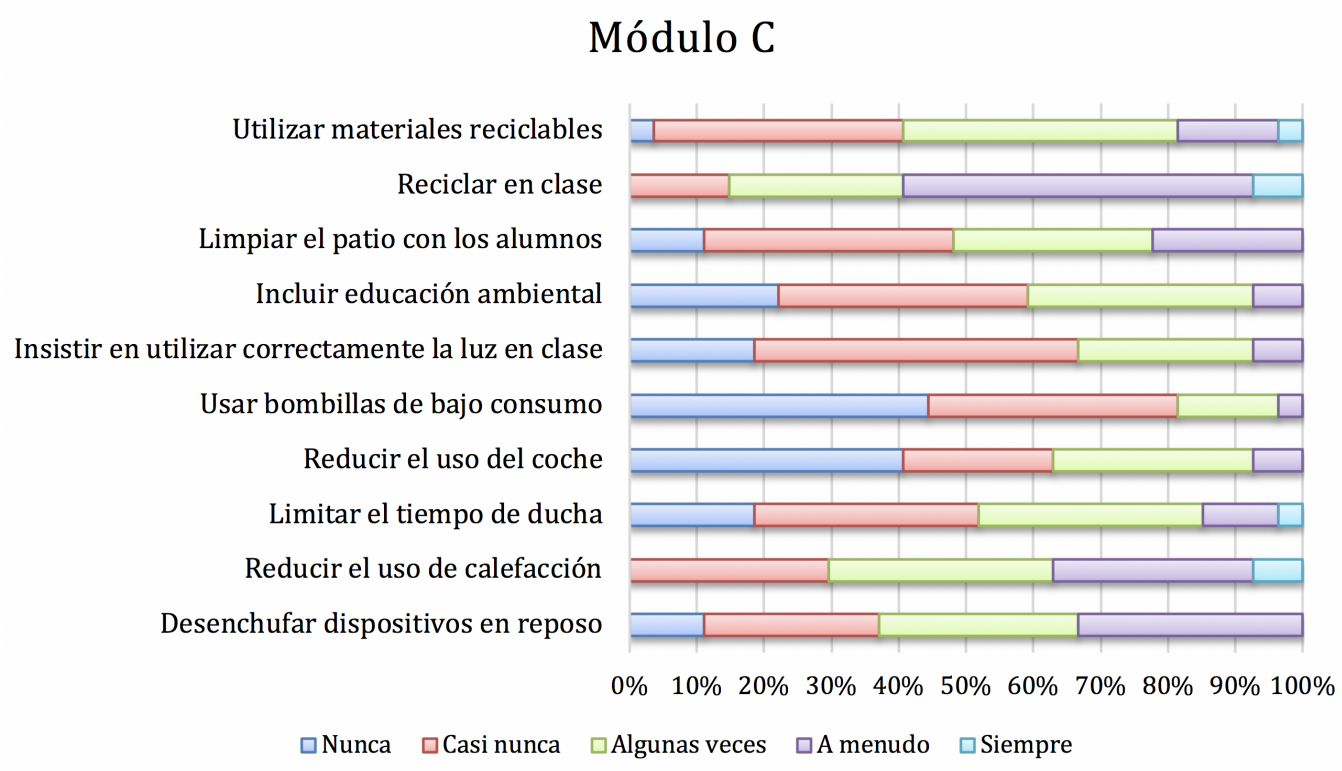

Figura 3. Registro de hábitos medioambientales de los docentes

A diferencia del anterior, este compendio de preguntas abarca de manera equitativa tanto ámbitos personales como educativos de los docentes, en relación con hábitos o costumbres (ver figura 3). 
A simple vista puede apreciarse una diferencia significativa: los cinco primeros valores revelan una mayor incidencia en el valor «Nunca» que en los cinco posteriores. Esto puede implicar una mayor preocupación por parte de los docentes por su transmisión de valores acordes con el cuidado del medioambiente a sus alumnos que por practicar hábitos de vida personal que vayan acorde a estos ideales. En este ámbito íntimo los encuestados manifestaron un afán mayor por cuidar aquellos aspectos que se combinan con lo económico y que no suponen un impacto en su comodidad (ver figura 4).

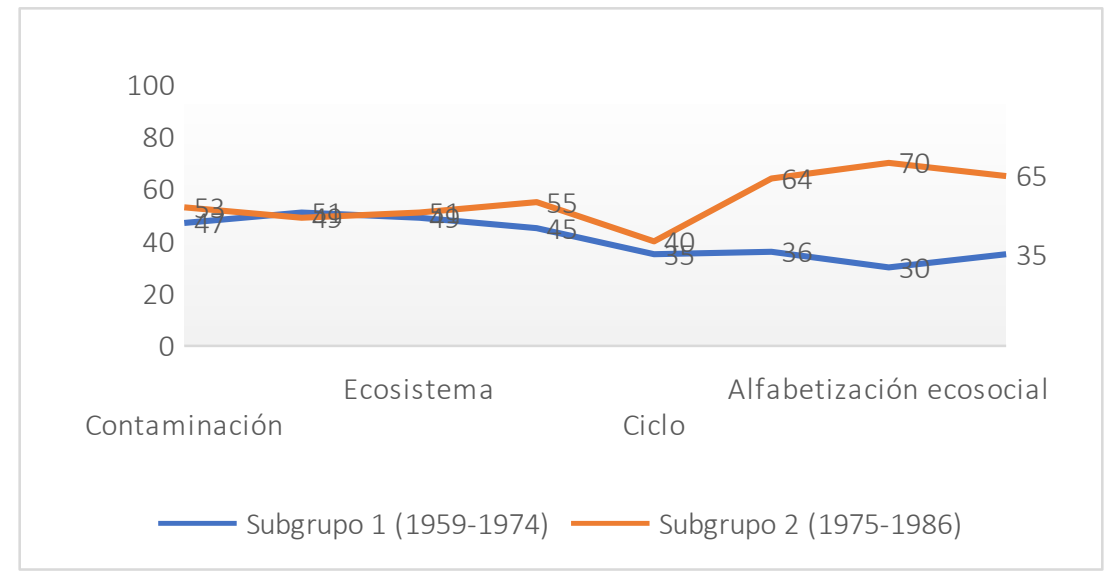

Figura 4. Relación porcentual de dos subgrupos en función de su edad y conocimiento acerca de conceptos medioambientales

En cuanto a sus actuaciones dentro del contexto académico, las opiniones reflejadas en la herramienta de evaluación parecen indicar que las actividades más comunes son las de «reciclar en clase» y «utilizar materiales reutilizables» (ver figura 6). Esta cercanía con lo pragmático y lo cotidiano confronta con que el valor donde más se señala «Nunca», como es el de «incluir educación ambiental en la programación». Es decir, los docentes llevan a cabo acciones que pueden considerarse propias de un currículum oculto donde se da importancia a este tipo de conceptos y se trabajan dentro del proceso enseñanza-aprendizaje, pero no se incluyen en la programación formal.

De igual manera que con el módulo B, una mirada más analítica de los datos recogidos puede ser útil a la hora de relacionar la edad del docente con su preocupación acerca de la educación ambiental. Por ello, se presenta la posible conexión entre estos factores de manera gráfica, teniendo en cuenta los mismos subgrupos y criterios que en la figura 5:

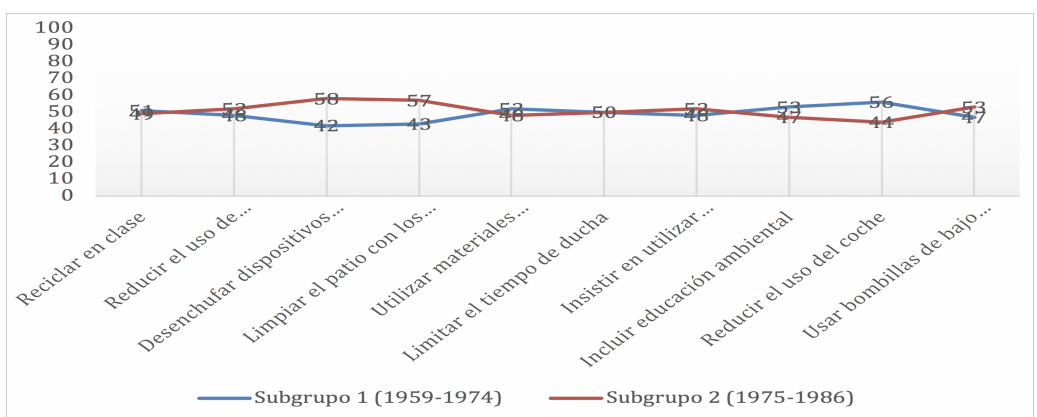

Figura 5. Relación porcentual de dos subgrupos en función de su edad y hábitos de cuidado del medioambiente en su vida personal y laboral. 


\section{Análisis de la tendencia observable tras la intervención didáctica}

Siguiendo esta línea, la intervención con los alumnos pretendía tanto introducir en su vida académica el concepto de EC para mejorar el nivel de sensibilización medioambiental como medir el impacto de una herramienta pedagógica sobre un hábito cotidiano relacionado con sus costumbres en el centro educativo.

Durante la intervención pedagógica en el aula del segundo ciclo de Educación Infantil, se llevó a cabo una recogida de datos diaria en la que cada niño podía traer o no papel albal como envoltorio. Trabajamos este concepto a través de las asambleas, utilizando sus nociones y sus pensamientos para comenzar el proceso de sensibilización.

De esta forma, la representación gráfica de esta herramienta estará constituida por una gráfica individual para cada alumno, de manera que pueda apreciarse si existe una tendencia negativa, positiva o neutra respecto al uso de este material, a fin de conseguir un objetivo final. La nomenclatura utilizada para los niños y niñas de la clase será la de «Alumno» y el número correspondiente, mientras que los datos se incluirán de tal forma que el valor numérico «1» corresponderá con un día en que el alumno sí ha utilizado el papel albal como envoltorio, mientras que si el valor numérico es 0 , implicará que ese día, el envoltorio habrá sido reutilizable y lavable. Si existe una falta de asistencia de ese alumno, la gráfica no reflejará ningún valor. En el eje horizontal se representan los días. Por lo tanto, los datos obtenidos son los siguientes:
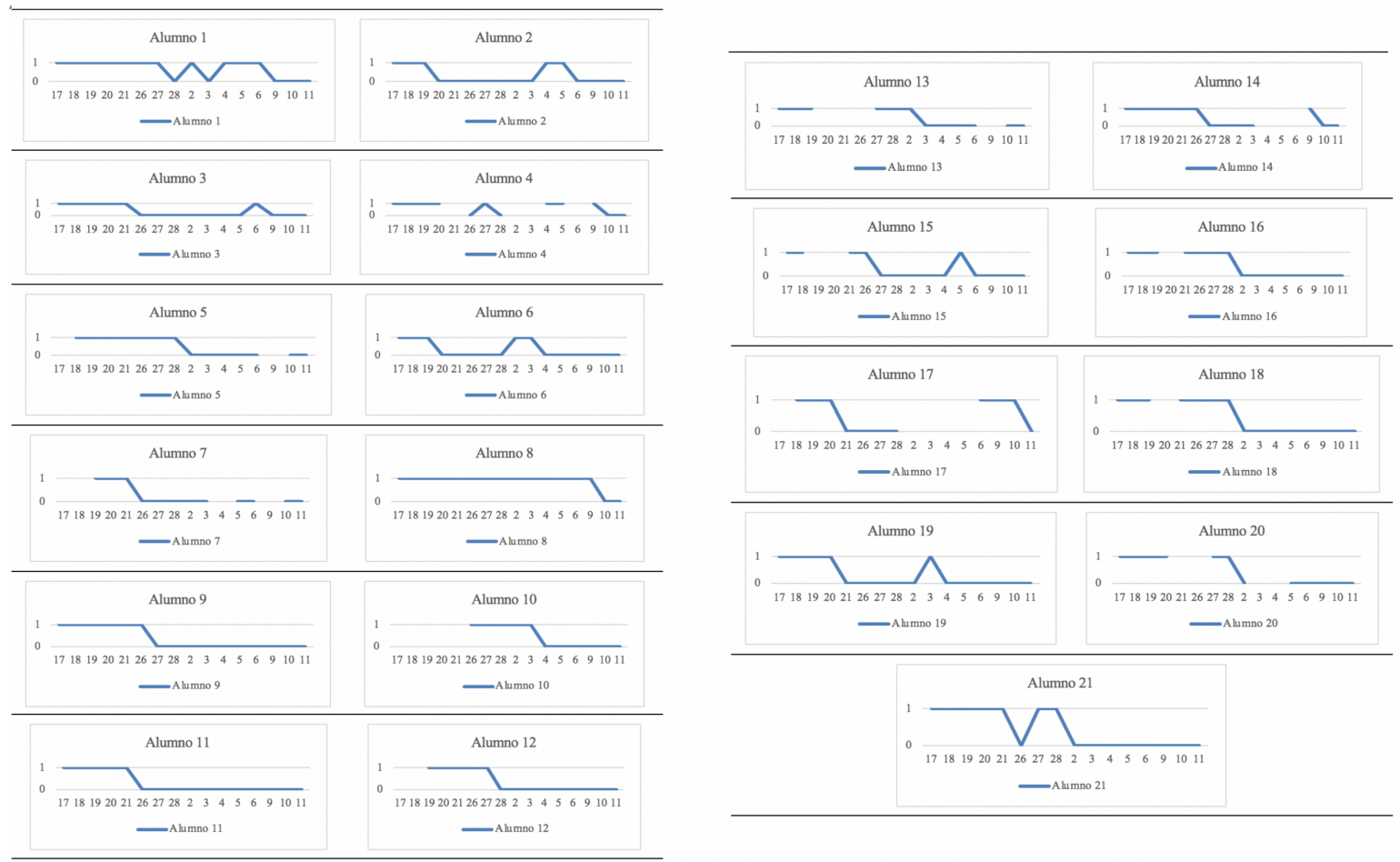

Figura 6. Tendencia observable de los niños ante la acción ambiental

Podemos observar cómo existe una ligera tendencia observable donde, tras el trabajo previo, la utilización de papel albal como envoltorio parece disminuir.

Esta tendencia puede medirse a través de la observación de distintos factores. Por ejemplo, se observa cómo un $66 \%$ de los alumnos terminaban la última semana sustituyendo el papel albal por otro tipo de envoltorio más apropiado. Además, 
teniendo en cuenta que, si contamos que cada alumno tiene cada día una oportunidad para traer o no traer papel albal, y restamos sus faltas de asistencias, el cambio de costumbre es ligeramente superior, llegando hasta el 54\%. Esta diferencia es significativa, puesto que no implica que los valores sean parejos, sino que se establece un nuevo hábito, que acaba imponiéndose sobre el anterior.

Este nuevo hábito se hace aún más patente si hacemos un sesgo temporal en el análisis. Si dividimos el proceso a la mitad, podemos observar que, durante la primera mitad, que comprende desde el 17 de febrero hasta el 28 de ese mismo mes, solo el $26 \%$ de las ocasiones posibles los alumnos sustituyeron el papel albal por otro tipo de envoltorio; mientras que, durante la segunda mitad, comprendida entre el 2 y el 11 de marzo, este $26 \%$ cambia drásticamente hasta llegar al $82 \%$, por lo que la tendencia al cambio es altamente significativa.

No obstante, pueden existir ciertas trabas para conseguir suscitar este cambio. En los datos obtenidos se refleja que un $62 \%$ de los alumnos han faltado en alguna ocasión durante el período de la intervención. Este hecho afecta irremediablemente a la tendencia positiva que puedan haber obtenido los alumnos hasta ese momento. Consecuentemente, se pueden extraer referencias de estos gráficos, ya que, una vez que se analizan los períodos de falta de asistencia de los alumnos, así como la longitud del mismo y las respuestas de los días posteriores a su vuelta, se puede establecer una media que arroja que si un alumno falta lo hará durante una media de 2 días, y esto afectará en su tendencia de manera negativa, puesto que la evidencia muestra que tras un período de ausencia, la tendencia se inclina hacia volver a usar el papel albal durante al menos 1,6 días. Es decir, si el trabajo en el aula se ve interrumpido, un alumno puede olvidar el concepto y la imposibilidad de seguir la dinámica puede afectarle sobre todo durante el día de su vuelta y el siguiente.

\section{Conclusiones y discusión}

\section{Discusión e inferencias}

Tras haber extraído y analizado los datos que se derivan de una intervención, resulta imprescindible llevar a cabo un proceso en el que relacionar ciertas ideas que surgen al combinar la teoría y la praxis.

Si atendemos a todo el trabajo realizado con los docentes, podemos observar que existe una diferencia evidente en el dominio de conocimientos complejos sobre medioambiente entre el grupo de docentes más jóvenes y el de los más veteranos, siendo los primeros más dominantes en este ámbito. No obstante, se aprecia al mismo tiempo que en los ámbitos personales y pedagógicos ambos grupos actúan de manera similar, por lo que puede inferirse que, a pesar de existir un dominio claro sobre los conceptos, la aplicación de los mismos sigue influenciada por las técnicas tradicionales (Chiu-Cheang, et al., 2019). Los docentes más jóvenes no han tenido acceso a una educación ambiental completa ni de calidad, e incluso ni siquiera durante su presente desempeño laboral encuentran que el currículum otorgue importancia a estos contenidos, por lo que su preocupación queda más relegada a aprendizajes más formales (Sumter, Bakker, Balkenende, Ruud y De Koning, 2019).

En cuanto a la intervención con los alumnos, los datos reflejan que existe una clara tendencia a la baja en la utilización de papel albal. El trabajo en el aula, unido al papel 
que juega la influencia del conjunto sobre el individuo, permite que cada niño vaya tomando conciencia de la importancia de realizar esta acción. El alumno observa cómo una pequeña tarea propia puede repercutir en el bien de la comunidad, la cual, además, le influye a la hora de realizar este gesto mediante una retroalimentación positiva.

Es en este contexto donde el niño puede aprender y ver modificadas ciertas conductas para conseguir un aprendizaje favorable, por lo que, uno de los mayores problemas a la hora de tratar de establecer un hábito en el aula son las faltas de asistencia. Los datos también reflejan su influencia, y puede deducirse que la interrupción de la influencia del docente y del ejemplo de sus iguales llevan al niño a que, en un ambiente que puede ser menos motivador desde el punto de vista pedagógico, olvide la actividad y su importancia. Por ende, puede resultar lógico que el trabajo sea extensible al ámbito familiar, a fin de que el efecto se viese ampliado y se paliase este problema.

Además, una de las principales ventajas observadas respecto a la educación ambiental es el término «economía circular», dado que casa a la perfección con el ritmo del colegio (Chen, Zhu, Xu y Meng, 2019). En concreto, el centro en el que se llevó a cabo la intervención se encuentra dentro de un plan llamado «Ecoescuelas». Por ello, la EC funciona en este contexto como sistema de reutilización dentro de una vida comunitaria donde los materiales son diseñados con un cuidado y una finalidad previa.

\section{Conclusiones}

A partir de los resultados obtenidos podemos declarar que existen claras evidencias de la consecución de los objetivos planteados. Así, puede concluirse que los docentes tienen un mayor dominio de la teoría cuanto más jóvenes son, y se considera el nivel medio como aceptable, puesto que solo en los términos más complejos existe un desconocimiento completo (Molano-Niño, 2012). Y en cuanto al concepto de la EC dentro del aula, cuya adquisición se basa en los principios de este modelo, son fácilmente adaptables a un aula, como se demuestra en la actividad llevada a cabo. Así, el último objetivo resultó en una propuesta atractiva y efectiva, puesto que la reacción de los alumnos fue positiva y la tendencia se midió de manera eficiente.

Por último, pueden extraerse ciertas conclusiones que la evidencia muestra, como por ejemplo:

- La educación ambiental resulta motivadora para los alumnos, ya que se refuerza el sentido del aula como conjunto, puesto que en la intervención se observa cómo la influencia de unos niños sobre otros ayuda a conseguir resultados positivos.

- El trabajo de conceptos relativos al medioambiente resulta eficiente puesto que el cambio de tendencia es innegable. El corto período de tiempo que duró la intervención fue suficiente para establecer que existe una propensión clara y beneficiosa.

- El cuerpo docente está dotado de un espectro amplio de conocimiento de conceptos pero su puesta en práctica es aún pobre debido a que la concienciación no es suficiente, ya que incluso ni los hábitos en su vida 
personal ni las actividades que llevan a cabo en sus aulas reflejan esta sensibilización.

A lo largo de la investigación se han podido observar claras evidencias de cómo una intervención integral podría ayudar más aún a una sensibilización y conocimiento en torno a temáticas sobre el medio ambiente y, en especial, sobre la economía circular.

Con todo ello, podemos declarar algunas de las líneas y propuestas que puedan mejorar el planteamiento desarrollado en esta primera intervención, como son:

1. Estudiar la influencia de una intervención dedicada a los docentes sobre educación ambiental donde realizar una encuesta inicial y una final para evaluar el posible cambio.

2. Realizar una ampliación temporal de la propuesta didáctica con los alumnos, donde evidenciar en mayor medida la tendencia observable.

3. Elaborar una programación basada en la educación ambiental cuyo impacto sea medido respecto a una programación estándar.

\section{Agradecimientos}

La génesis de este trabajo se remonta al mes de septiembre de 2019, siendo parte del proyecto «CienciaCreActiva: Científicas en la economía circular» financiado por la Fundación Española para la Ciencia y la Tecnología (FECYT) (FCT-18-13150) al que se puede acceder a través de su página web: https://cienciacreactiva.bio3project.es

\section{Referencias}

Albert, M. J. (2007). La Investigación Educativa. Claves Teóricas. Madrid: McGraw-Hill/ Interamericana de España, S. A. U.

Bebbington, J., Russell, S., y Thomson, I. (2017). Accounting and sustainable development: Reflections and propositions. Critical Perspectives on Accounting, 48, 21-34. https://doi.org/10.1016/j.cpa.2017.06.002.

Boudes, Ph. (2011). United Nations Conference on the Human Environment. In J. Newman (ed.) Green Ethics and Philosophy-The Green Series: Toward a Sustainable Environment, Vol. VIII (pp. 410-413) Sage Publications Inc.

Boyd, W. (2020). 'Nothing Goes to Waste': A professional learning programme for early childhood centres. Australasian Journal of Early Childhood, 45(1), 69-81. https://doi.org/10.1177/1836939119885313.

Buil, P., Roger-Loppacher, O., y Tintoré, M. (2019). Creating the Habit of Recycling in Early Childhood: A Sustainable Practice in Spain. Sustainability, 11(22), 6393. https://doi.org/10.3390/su11226393.

Campos y Covarrubias, G., y Lule Martínez, N. E. (2012). La observación, un método para el estudio de la realidad. Xhimai, 7(13), 45-60. https://bit.ly/3dyNtOx.

Chen, K., Zhu, K., Xu, J., y Meng, M. (2019). Sustainable Development Education from the Perspective of China. ICIEI 2019: Proceedings of the 2019. 4th International Conference on Information and Education Innovations. Durham, United Kingdom. New York, United States: Association for Computing Machinery https://bit.ly/2Y0yrdZ. 
Ghisellini, P., y Ulgiati, S. (2019). Managing the transition to the circular economy. In M. Brandao, D. Lazarevic, y G. Finnveden, (Eds.), Handbook of the circular economy. Edward Elgar Publishing Ltd. https://bit.ly/3ePr2oF.

Chiu-Cheang, C., Yan-Cheung, T., Wing-Mui-So, W., Nga-Yee-Cheng, I., Fok, L., Ho-Yeung, C., y Fai-Chow, C. (2019). Enhacing pupils' proenvironmental knowledge, attitudes and behaviors on plastic recycling education: A quasi-experimental study in primary schools. In W. Wing Mui So, C. Fai Chow, y J. Chi Kin Lee, (Eds.), Environmental Sustainability and Education for Waste Management (pp. 159188). Springer. https://doi.org/10.1007/978-981-13-9173-6_10.

Comisión Europea (2013). Libro Verde. Un marco para las políticas de clima y energía en 2030. https://cutt.ly/WyLxwZM.

Fundación para la Economía circular (marzo 2017). Por qué y cómo desarrollar estrategias de economía circular en el ámbito regional. Madrid. https://goo.gl/YcPKnK.

Hernández, R., Fernández, C., y Baptista, M. P. (2010). Metodología de la investigación (5 a ed.). Madrid: Mc Graw-Hill Interamericana Editores.

Irving-Olsen, S. (2019). The Long Road to a Circular Economy. Integrated Environmental Assessment and Management, 15(4), 492-493. https://doi.org/10.1002/ieam.4170.

Latorre, A., del Rincón, D., y Arnal, J. (2003). Bases metodológicas de la investigación educativa. Barcelona: Ediciones Experiencia.

Lee, J. C. K. (2000). Teacher receptivity to curriculum change in the implementation stage: The case of environmental education in Hong Kong. Journal of Curriculum Studies, 32(1), 95-115. https://doi.org/10.1080/002202700182871.

Leff, E. (s.f.). Globalización, Racionalidad Ambiental y Desarrollo Sustentable. https://cutt.ly/wd8Zxfa.

Leff, E. (2004). La racionalidad ambiental. México: Siglo XXI Editores.

Molano-Niño, A. B. (2012). La complejidad de la educación ambiental: Una mirada desde los siete saberes necesarios para la educación del futuro de Morin. Revista de Didáctica Ambiental, 8(11), 1-9. https://bit.ly/2Xy9hEu.

Morató, J., Tollin, N., Jiménez, L., Villanueva, B., Plá, M., Betancourth, C., de la Cruz, J. L., y Pérez-Lagüela, E. (2017). Situación y evolución de la economía circular en España. Madrid: Fundación COTEC para la Innovación. https://bit.ly/3eRgoNY.

Naciones Unidas (1973). Informe de la Conferencia de las Naciones Unidas sobre el Medio Humano. Estocolmo, 5 a 1 de junio de 1972. New York, United States: Naciones Unidas. Recuperado de https://cutt.ly/gdEBULF.

Pearce, D. W., y Turner, R. K. (1990). Economics of natural resources and the environment. Londres: Harvester Wheatsheaf.

Pearson, P. J. G. (2019). Energy transitions. En M. Vernengo, E. Perez, y B. J. Rosser Jr. (Eds.), The New Palgrave Dictionary of Economics. Living edition (pp. 1-24). 
London: Palgrave Macmillan. https://doi.org/10.1057/978-1-349-951215_3025-1.

de Canales, F. H., de Alvarado, E. L., y Pineda, E. B. (1994). Metodología de la investigación: Manual para el desarrollo de personal de salud. Washington: Organización Panamericana de la Salud. https://bit.ly/2Y597nd.

Ramírez-Treviño, A., Sánchez-Núñez, J. M., y García-Camacho, A. (2004). El Desarrollo Sustentable: Interpretación y Análisis. Revista del Centro de Investigación. Universidad La Salle, 6(21), 55-59. https://bit.ly/2XwVoXa.

Rekalde, I., Vizcarra, M. T., y Macazaga, A. M. (2014). La observación como estrategia de investigación para construir contextos de aprendizajes y fomentar procesos participativos. Educación XX1, 17(1), 201-220. https://bit.ly/378YSm0.

Sánchez-Emeterio, G., y Figueira, C. (2019). Eco-social literacy: Circular economy conceptions in initial teacher training. Proceedings of the 9th International Conference the Future of Education (pp. 486-490). Bologna, Italy: Filodiritto Publisher.

Schoden, F., Siebert, A., Keskin, A., Herzig, K., Straus, M., y Schwenzfeier-Hellkamp, E. (2020). Building a Wind Power Plant from Scrap and Raising Public Awareness for Renewable Energy Technology in a Circular Economy. Sustainability, 12, 90. https://doi.org/10.3390/su12010090.

Stahel, W., y Reday-Mulvey, G. (1977). The potential for substituting manpower for energy. Geneva, Switzerland: Geneva Research Centre. https://bit.ly/2MvHdLQ.

Stahel, W., y Reday, G. (1981). Jobs for Tomorrow, the Potential for Substituting Manpower for Energy. New York: Vantage Press.

Sumter, D., Bakker, C. A., Balkenende, Ruud, B., y De Koning, J. (2019). Design competencies for a circular economy. Product Lifetimes and The Environment. 3, 1-6. https://bit.ly/3gRGnH5.

Taylor, S. J., y Bogdan, R. (1984). Introducción a los métodos cualitativos de investigación. La búsqueda de significados. Barcelona: Paidós. https://cutt.ly/xgKwAab.

Toro-Sánchez, F. J. (2007). El desarrollo sostenible: Un concepto de interés para la geografía. Cuadernos geográficos, 40(1), 149-181. https://bit.ly/3ePUk6x.

Torres, M. (1996). La dimensión ambiental: Un reto para la educación de la nueva sociedad. Interlínea Editores Ltda. https://bit.ly/2MvqDvi.

Upadhayay, S., y Alqassimi, 0. (2019). Transition from Linear to Circular Economy. 2, 62-74. https://bit.ly/308lotE. 


\section{Anexo I}

\section{Cuestionario}

Las preguntas que se presentan forman parte de un proyecto de Economía Circular de la Universidad de Castilla la Mancha con la finalidad de conocer las conductas, creencias y emociones relacionadas con el cuidado del medioambiente. Por tanto, no existen respuestas correctas o incorrectas. Además, es totalmente anónimo. Por ello, te pedimos que se complete este documento con la mayor sinceridad posible. La información recogida será tratada solo con fines de formación o investigación en la Universidad de Castilla-La Mancha.

- Género:

Módulo A

- Año de nacimiento:

- Estudios realizados:

- Nivel:

- $\quad$ Años de experiencia:

Módulo B

En una escala de 1 a 5 en la que 1 significa que "no estás NADA preocupado por el medio ambiente" y el 5 que "estás MUY preocupado/a", ¿en qué casilla te colocarías a nivel general?

\begin{tabular}{|c|c|c|c|c|}
\hline preocupado/a, ¿en qué casilla te colocarías a nivel general? & 3 & 4 & 5 \\
\hline 1 & 2 & & & \\
\hline
\end{tabular}

\begin{tabular}{|c|c|c|c|c|c|}
\hline \multicolumn{7}{|c|}{ Indica con una X el grado de conocimiento de los siguientes conceptos } \\
\hline & $\begin{array}{c}\text { Lo desconozco } \\
\text { totalmente }\end{array}$ & $\begin{array}{c}\text { Lo desconozco } \\
\text { bastante }\end{array}$ & $\begin{array}{c}\text { Lo conozco } \\
\text { un poco }\end{array}$ & $\begin{array}{c}\text { Lo conozco } \\
\text { bastante }\end{array}$ & $\begin{array}{c}\text { Lo conozco } \\
\text { perfectamente }\end{array}$ \\
\hline Economía circular & & & & & \\
\hline Ecosistema & & & & & \\
\hline Ciclo & & & & & \\
\hline Modelo lineal & & & & & \\
\hline Desarrollo sostenible & & & & & \\
\hline Sobreexplotación & & & & & \\
\hline Contaminación & & & & & \\
\hline Alfabetización ecosocial & & & & & \\
\hline
\end{tabular}

Módulo C

\begin{tabular}{|l|l|l|l|l|l|}
\hline \multicolumn{5}{|c|}{ ¿Con qué frecuencia realizas las siguientes conductas? Marca con una X } \\
\hline $\begin{array}{l}\text { Desenchufar los dispositivos } \\
\text { conectados en reposo }\end{array}$ & Nunca (1) & Casi nunca (2) & $\begin{array}{c}\text { Algunas veces } \\
\text { (3) }\end{array}$ & A menudo (4) & Siempre (5) \\
\hline $\begin{array}{l}\text { Reducir la calefacción para } \\
\text { disminuir el gasto energético }\end{array}$ & & & & \\
\hline $\begin{array}{l}\text { Limitar el tiempo de ducha } \\
\text { para ahorrar agua }\end{array}$ & & & & & \\
\hline $\begin{array}{l}\text { No utilizar el coche por } \\
\text { razones medioambientales }\end{array}$ & & & & & \\
\hline $\begin{array}{l}\text { Usar bombillas de bajo } \\
\text { consumo }\end{array}$ & & & & & \\
\hline $\begin{array}{l}\text { Insistir en utilizar } \\
\text { correctamente la luz en clase }\end{array}$ & & & & & \\
\hline $\begin{array}{l}\text { Incluir educación } \\
\text { medioambiental en la } \\
\text { programación }\end{array}$ & & & & & \\
\hline $\begin{array}{l}\text { Limpiar el patio con los } \\
\text { alumnos }\end{array}$ & & & & & \\
\hline Reciclar en clase & & & & \\
\hline $\begin{array}{l}\text { Utilizar materiales reciclables } \\
\text { en las actividades }\end{array}$ & & & & & \\
\hline
\end{tabular}




\section{Anexo II. Registro de observación diario}

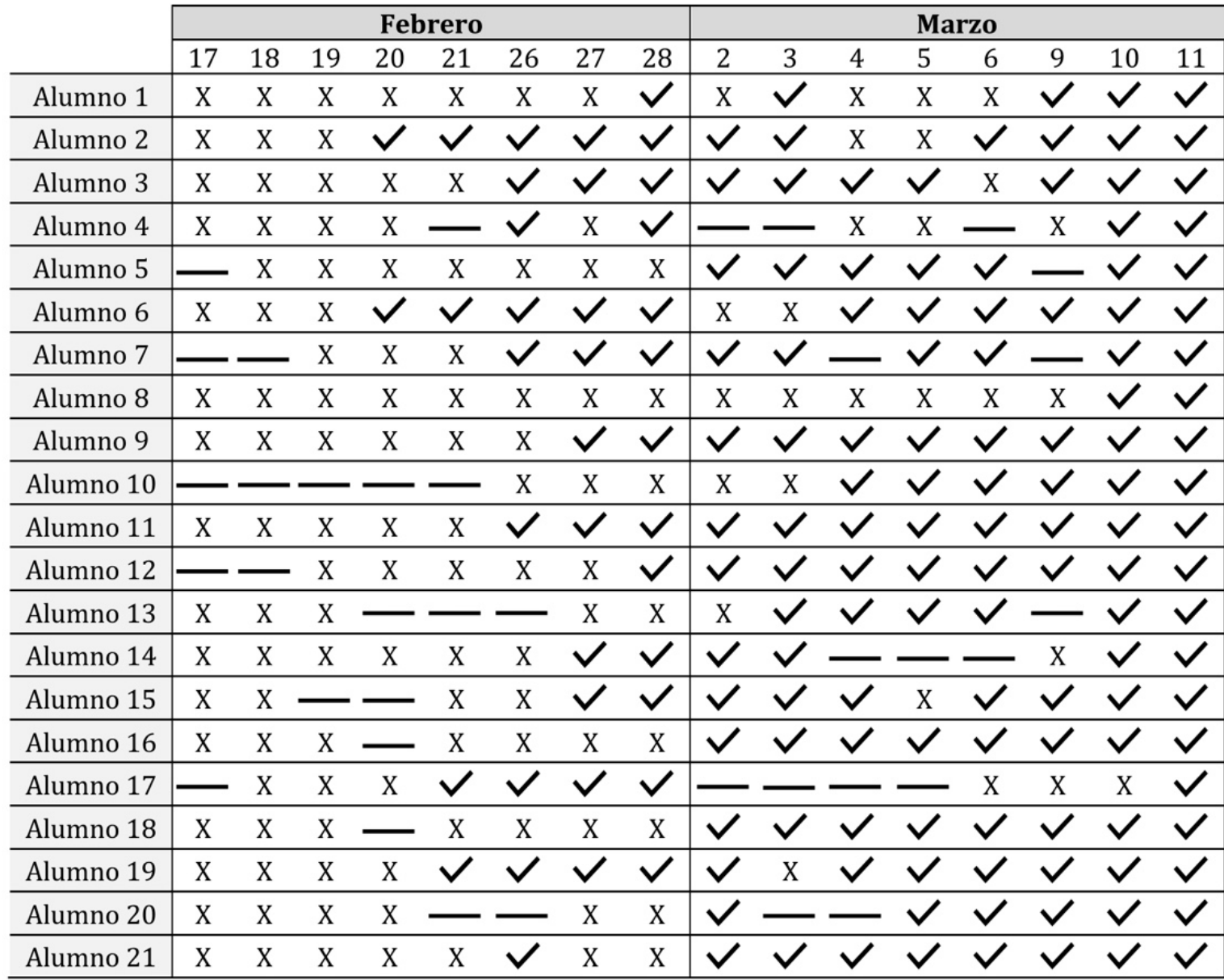

